\title{
Effect of Carcinogen 1,2-Dimethylhydrazine Treatment on Fiber Types in Skeletal Muscles of Male Wistar Rats
}

\author{
V. SMERDU ${ }^{1}$, M. PERŠE ${ }^{2}$ \\ ${ }^{1}$ Institute of Anatomy, Faculty of Medicine, University of Ljubljana, Ljubljana, Slovenia, ${ }^{2}$ Institute \\ of Pathology, Medical Experimental Centre, Faculty of Medicine, University of Ljubljana, \\ Ljubljana, Slovenia
}

Received October 11, 2016

Accepted February 27, 2017

On-line July 18, 2017

\section{Summary}

The cancerogen 1,2-dimethylhydrazine (DMH), widely used in the experimental animal model of carcinogenesis, affects various organs, but its effect on muscle fibers is unknown. To evaluate the effect of 15-week DMH treatment on the fiber size and myosin heavy chain (MyHC) isoforms, which substantially determine fiber types and their contractile characteristics, pure and hybrid fiber types were immunohistochemically determined according to the MyHC isoform expression in soleus, extensor digitorum longus, gastrocnemius medialis and lateralis muscles of DMH-treated and control male Wistar rats. Whereas the size of fibers was mostly unaffected, the MyHC isoform expression was partially affected in both gastrocnemius samples, but not in the soleus and extensor digitorum longus of DMH-treated rats. The lower proportions of hybrid fiber types and especially that of type $1 / 2 x$ in most gastrocnemius samples of $\mathrm{DMH}$-treated rats resulted in a shift towards a single $\mathrm{MyHC}$ isoform expression, but the extent and pattern of the MyHC isoform shift varied across the different gastrocnemius samples. Such variable response to DMH treatment across muscles indicates that each muscle possesses its own adaptive range. These findings are essential for an accurate evaluation of skeletal muscle characteristics in DMH animal model.

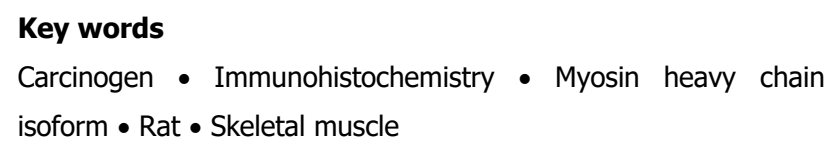

\section{Corresponding author}

V. Smerdu, Institute of Anatomy, Faculty of Medicine, University of Ljubljana, Korytkova 2, 1000 Ljubljana, Slovenia. E-mail: vika.smerdu@mf.uni-lj.si

\section{Introduction}

The 1,2-dimethylhydrazine (DMH) or azoxymethane (AOM) model is a well-established and widely used model of experimental colon carcinogenesis (Corpet and Pierre 2005). DMH and its metabolite AOM are carcinogens that induce multistep development of colon carcinogenesis in rodents (Perše and Cerar 2011). The susceptibility for DMH/AOMinduced colorectal carcinogenesis is species-, strain-, sex- and age-dependent (Kobaek-Larsen et al. 2000). The stage and the number of the colonic lesions depend on the total amount of the carcinogen administered and the latency period. In long-term studies DMH is administered weekly for 15-20 weeks in a relatively low concentration $(20 \mathrm{mg} / \mathrm{kg})$, and 6-10 weeks after the last application the animals are scored for all the steps of colonic lesions (dysplastic crypts, adenomas, adenocarcinomas) (for review see Perse and Cerar 2005, Perše and Cerar 2011). At that point colonic lesions are small enough that do not affect the animal's health status but nonetheless make it possible to evaluate the effect of the tested factor on the multistep development of colon carcinogenesis (Bruce 2003). In our laboratory the effect of various factors on $\mathrm{DMH}$-induced colon carcinogenesis is usually evaluated 6 weeks after the last application of DMH $(20 \mathrm{mg} / \mathrm{kg}$ per week for 15 weeks) (Perse et al. 2009, Perše et al. 2012).

Although DMH and AOM are considered highly specific colon carcinogens, they affect other organ systems as well, like the liver (Netto et al. 1992, Perse et 
al. 2009) and may cause tumors of the Zymbal glands (i.e. modified sebaceous glands at the base of external auditory canal) in a small percentage of animals (Shetye et al. 1994). Further, it was reported that the oxidative status in the circulation (Devasena et al. 2006) and heart (Perse et al. 2009) were affected. Moreover, in a mouse DMH model it was shown that the catabolism and energy production of skeletal muscle were affected by a highmobility group box 1 protein, released by tumor cells. This protein not only increased the proliferation of tumor cells, but it also activated muscle to supply glutamine as an energy source to cancer cells (Luo et al. 2014).

Skeletal muscles are known to be susceptible to various factors, such as a changed pattern of neural discharge, muscle activity, loading and hormonal status. Since the skeletal muscles are not only a locomotor organ, but are the metabolic engine of the body and have immunogenic and endocrine functions as well (Nielsen and Pedersen 2008, Pedersen et al. 2007), it would be of a great importance for all the studies that employ this experimental model to evaluate which skeletal muscle components are affected by $\mathrm{DMH}$ treatment. As the metabolism, size and contractile characteristic of muscle fibers are interrelated, we postulated that not only metabolism (Luo et al. 2014) but muscle fibers could be affected by DMH treatment as well. However, the effect of DMH treatment on the muscle fiber characteristics has not yet been studied.

Skeletal muscles can adapt to changed conditions with a shift in the expression of myosin heavy chain (MyHC) isoforms, which are one of the most relevant markers of fiber types and their contractile characteristics (Baldwin and Haddad 2001, Schiaffino et al. 2013). There are four major MyHC isoforms that determine slow or type 1 fibers and fast type $2 \mathrm{a}, 2 \mathrm{x}(2 \mathrm{~d})$ and $2 \mathrm{~b}$ fibers in rat skeletal muscles (Pette and Staron 1997, DeNardi et al. 1993). In addition to the major four pure fiber types, hybrid fibers co-expressing mainly two MyHC isoforms are present as well (Talmadge 2000, Baldwin and Haddad 2001, Caiozzo et al. 2003). The shift in MyHC isoform expression leads to fiber type transitions (Pette and Staron 1997, Baldwin and Haddad 2001, Schiaffino et al. 2013). Beside the MyHC expression even the size of fibers can be modulated by various factors. Namely, resistance training, overload and certain humoral factors (androgens, $\quad \beta$-agonists, IGF-1) induce fiber hypertrophy, whereas decreased activity or disuse, denervation, decreased loading and increased level of glucocorticoids result in fiber atrophy (Blaauw et al. 2013).

Since the DMH animal model is frequently used in dietary and exercise studies (Perse et al. 2009, Perše et al. 2012), it is essential to evaluate the potential effect of DMH treatment on skeletal muscle fibers for an accurate and valid interpretation of the results. The aim of this study was thus to evaluate the effect of long-term $\mathrm{DMH}$ treatment on skeletal muscles by analyzing the expression of MyHC isoforms immunohistochemically and the fiber type size morphometrically. Since each muscle has its own characteristic range of possible extent of fiber type transitions, assumed to be related not only to the muscle intrinsic myogenic or genetic constraints (Schiaffino and Reggiani 2011) but also sex-dependent (Drzymala-Celichowska et al. 2012), various hind limb muscles of male rats were analyzed.

\section{Materials and Methods}

\section{Animals and experimental protocol}

Male Wistar (HsdRccHan ${ }^{\mathrm{TM}}$ : WIST) rats were used in the study. They were acclimatized and housed at Medical Experimental Centre (Ljubljana, Slovenia) 4 per cage $\left(1,825 \mathrm{~cm}^{2}\right.$ floor space) on Lignocel $3 / 4$ bedding material (Germany) at $22-23{ }^{\circ} \mathrm{C}$ and $55 \pm 10 \%$ humidity, $12 \mathrm{~h} \mathrm{light/dark}$ cycle (illumination between 07:00 p.m. and 07:00 a.m.) and had free access to food (Altromin 1324, Germany) and tap water. Twelve-week-old rats were randomly divided into two groups, namely a DMH-treated $(n=6)$ group and an untreated control group $(n=5)$. The rats were treated either with $\mathrm{DMH}$ $(20 \mathrm{mg} / \mathrm{kg}$, dissolved in $0.001 \mathrm{M}$ EDTA; $\mathrm{pH}$ 6.8) or saline $(0.9 \% \mathrm{NaCl})$ instead of $\mathrm{DMH}$, both administered subcutaneously once a week for 15 consecutive weeks (Perse et al. 2009, Perše et al. 2012). The animals of both groups were euthanized 6 weeks after last DMH or saline treatment at age of 33 weeks. The care and use of animals was approved by the National Ethic Committee of the Republic of Slovenia (License No. 34401-61/2007/7) and conducted in accordance with the European Convention ETS 123, Directive 86/609/EEC regarding the protection of animals used for experimental and other scientific purposes and Slovenian legislation on the protection of animals used in research.

The body weight of the rats was recorded once a week, water and food intake was recorded three times 
per week during the entire experiment. Before and during the experiment blood was taken for measurement of red and white blood picture and serum lipid profile. Fecal corticosterone levels were measured during the experiment. At autopsy all internal organs, including abdominal fat were removed, weighed and macroscopically examined (Perse et al. 2009, Perše et al. 2012).

Muscle samples and MyHC isoform immunohistochemistry

After euthanasia, four skeletal muscles, known to differ in the fiber type proportions, were excised from the right hind limb of both experimental groups: slow soleus (SOL), fast extensor digitorum longus (EDL), and the heterogeneous gastrocnemius lateralis (GL) and medialis (GM) muscles. For the latter two muscles a non-random distribution of fiber types is known, with the prevalence of slower ones in the deep region and a gradual change to the superficial region with the predominance of faster ones, (Matsakas et al. 2006). GL and GM samples were therefore further divided into a superficial part (GLs, GMs) and a deep part (GLd, GMd), whereas the intermediate, mixed part of GL (GLi), containing more fast fiber types than GLd and less than GLs, was also separately analyzed. Whole muscle samples were frozen in liquid nitrogen and stored at $-80^{\circ} \mathrm{C}$ until being processed for immunohistochemistry.

Fiber types were determined according to the expression of $\mathrm{MyHC}$ isoforms demonstrated with a set of monoclonal antibodies specific to $\mathrm{MyHC}$ isoforms: BA-D5 (MyHC-1) and SC-71 (MyHC-2a), BF-F3 (MyHC-2b) (Schiaffino 1986), and 6H1 (MyHC-2x) (Lucas et al. 2000). The supernatants of BA-D5, SC-71, and BF-F3 antibodies were produced in the local laboratory from corresponding cell lines provided from Deutsche Sammlung von Mikroorganismen und Zellkulturen (DSMZ, Braunschweig, Germany), whereas that of $6 \mathrm{H} 1$ was purchased from the Developmental Studies Hybridoma Bank (DSHB, University of Iowa, USA).

Serial muscle cryosections $(10 \mu \mathrm{m})$ were preincubated in phosphate buffered saline, containing $0.5 \%$ bovine serum albumin (PBS/BSA) and rabbit serum (1:40), for $30 \mathrm{~min}$. The appropriate dilutions of antibodies with PBS/BSA were determined (BA-D5 1:500; SC-71 1:200; 6H1 1:50; BF-F3 1:20). Subsequently, the sections with primary antibody were incubated in a humidified box overnight at $4{ }^{\circ} \mathrm{C}$. As a control to each set of the analyzed samples, a slide with serial sections was simultaneously incubated in PBS/BSA, but without the primary antibody. The reactivity of the antibodies BA-D5, SC-71 and BF-F3 was revealed with horseradish peroxidase conjugated secondary antibody (P260, Dako), diluted (1:100) in PBS/BSA, containing rabbit serum (1:40). To reveal the secondary antibody binding, the sections were incubated in $0.05 \%$ diaminobenzidine tetrahydrochloride hydrate (DAB) and $0.01 \% \mathrm{H}_{2} \mathrm{O}_{2}$ in $0.2 \mathrm{M}$ acetate buffer ( $\mathrm{pH}$ 5.2) for approximately $7 \mathrm{~min}$ in the dark (Gorza 1990, Smerdu and Soukup 2008). The binding of $6 \mathrm{H} 1$ was demonstrated using NovoLink Polymer Detection System and following the instructions of the producer (Leica Biosystems, Newcastle, UK). The control sections were respectively incubated either with the secondary antibody or NovoLink Polymer Detection System.

\section{Muscle section analysis}

Serial muscle sections were analyzed using a computer-assisted system for image analysis, developed in our laboratory in the collaboration with colleagues from Academy of Sciences of the Czech Republic (Karen et al. 2009). Briefly, in each muscle section stained with different antibody specific to MyHC isoform, the same selected area was registered by a digital camera, connected to a microscope. Thereafter, the registered images were adjusted to each other (Muscle Reg program) and then on the average 170 fiber contours were outlined manually in one of the registered images using a program Ellipse (ViDiTo, Košice, Slovakia). The set of registered and adjusted images with the superimposed fiber contours was used as an input data to computer program (FibClasM program) to determine the labelling pattern of fibers with different antibodies. Finally, using several macros (Microsoft Excel), fiber types were determined according to the labelling pattern of fibers. In addition, the average diameters of fiber types were computed from the fiber contours.

\section{Statistics}

The proportions (\%) and the average diameters $(\mu \mathrm{m})$ of fiber types were determined within each muscle or muscle region (SOL, EDL, GMd, GMs, GLd, GLi, GLs) of control $(\mathrm{n}=5)$ and DMH-treated rats $(\mathrm{n}=6)$. 
Thereafter the mean proportion and average diameter of each fiber type was determined for each of the analyzed muscles of both groups. Descriptive statistics were used to calculate means, standard errors of the means, and the ranges of all variables. To determine whether the differences between the two groups were significant, the means of the same fiber type in the homonymous muscles of both groups were compared either by $t$-test or Mann-Whitney test, depending on the distribution of the standard deviations of means, which was determined by a Shapiro-Wilks test. The data are presented as a mean \pm standard error of the mean (SEM). The differences with $\mathrm{p}<0.05$ were considered as significant, whereas the differences with $p=0.05-0.1$ were considered as trends for differences. Data were analyzed using SPSS Statistics program Version 20.

\section{Results}

\section{Health status of animals}

The general health status, locomotion, behaviour, and body weight of DMH-treated rats did not differ from those of the control rats throughout the experiment. No colonic lesions were found in the control group, while all rats in the DMH-treated group developed small, microscopically visible dysplastic colon lesions (6.75 \pm 0.82 foci of dysplastic crypts, $0.5 \pm 0.27$ adenomas, $0.38 \pm 0.26$ adenocarcinomas). DMH-treated rats exhibited no signs of cancer-induced cachexia or muscle wasting (Perse et al. 2009, Perše et al. 2012).

The body weights of rats were comparable in both groups during the entire experiment, as well of food, calorie and water intake. Autopsy showed no significant differences in the relative weights of internal organs or abdominal fat between groups. No differences were found in the serum lipid profile (cholesterol, LDL, HDL, triglyceride), blood parameters, serum $\mathrm{LDH}$ levels, or corticosterone levels between both groups (Perse et al. 2009, Perše et al. 2012), demonstrating that the rats in both groups had comparable clinical and physical status.

\section{Fiber types}

Four pure fiber types, expressing a single MyHC isoform (1, 2a, 2x, or 2b), and hybrid fiber types, co-expressing two or even more MyHC isoforms were determined for each muscle sample (Figs 1 and 2). In the analyzed muscles 11 hybrid fiber types $(1 / 2 \mathrm{a}, 1 / 2 \mathrm{ax}$, $1 / 2 \mathrm{axb}, 1 / 2 \mathrm{x}, 1 / 2 \mathrm{xb}, 1 / 2 \mathrm{ab}, 1 / 2 \mathrm{~b}, 2 \mathrm{ax}, 2 \mathrm{ab}, 2 \mathrm{axb}, 2 \mathrm{xb})$ were determined according to the pattern of MyHC isoforms' co-expression. Some of the hybrid fiber types were very scarce and present only in few muscle samples (Table 1). In fact, all 11 hybrid fiber types were found only in the EDL of the control animals, whereas only hybrid types $2 \mathrm{xb}, 1 / 2 \mathrm{x}, 2 \mathrm{ax}$, and $1 / 2 \mathrm{a}$ were more frequent, present in more than one third of the analyzed muscle samples. Therefore, to reveal an eventual effect of DMH treatment on the muscle fibers, the average proportions and diameters of pure fiber types, four of the most numerous hybrid fiber types, $2 \mathrm{xb}, 1 / 2 \mathrm{x}, 2 \mathrm{ax}$, and $1 / 2 \mathrm{a}$, whose average proportion was above $2 \%$ and were present in the majority of muscle samples, and of pooled all hybrid fiber types $(\mathrm{H})$ of control and DMH-treated rats were compared (Figs 3 and 4).

Table 1. Range of the average proportion (\%) per muscle sample and frequency ( $n$ ) of individual hybrid fiber types in the analyzed muscle samples (totally 77 samples) of control and DMH-treated rats.

\begin{tabular}{|c|c|c|c|}
\hline $\begin{array}{l}\text { Range of the average } \\
\text { proportion/muscle sample }(\%)\end{array}$ & & Hybrid fiber type & \\
\hline $0-0.2$ & $1 / 2 \mathrm{axb}(\mathrm{n}=1 / 77)$ & $1 / 2 \mathrm{ab}(\mathrm{n}=4 / 77)$ & $1 / 2 b(n=4 / 77)$ \\
\hline $0-0.4$ & $1 / 2 \mathrm{ax}(\mathrm{n}=10 / 77)$ & $1 / 2 \times b(n=9 / 77)$ & \\
\hline $0-2.0$ & $2 \mathrm{ab}(\mathrm{n}=14 / 77)$ & $2 \mathrm{axb}(\mathrm{n}=12 / 77)$ & \\
\hline $0-8.0$ & $2 \mathrm{ax}^{*}(\mathrm{n}=53 / 77)$ & $1 / 2 \mathrm{a} *(\mathrm{n}=33 / 77)$ & \\
\hline $0-22.0$ & $2 \mathrm{xb}^{*}(\mathrm{n}=53 / 77)$ & $1 / 2 x *(n=33 / 77)$ & \\
\hline
\end{tabular}

$\mathrm{n}$ : number of samples in which an individual hybrid fiber type was present. *Note that type $2 x b, 1 / 2 x, 2 a x$ and $1 / 2 a$ fibers were the most frequent hybrid fiber types. 
control

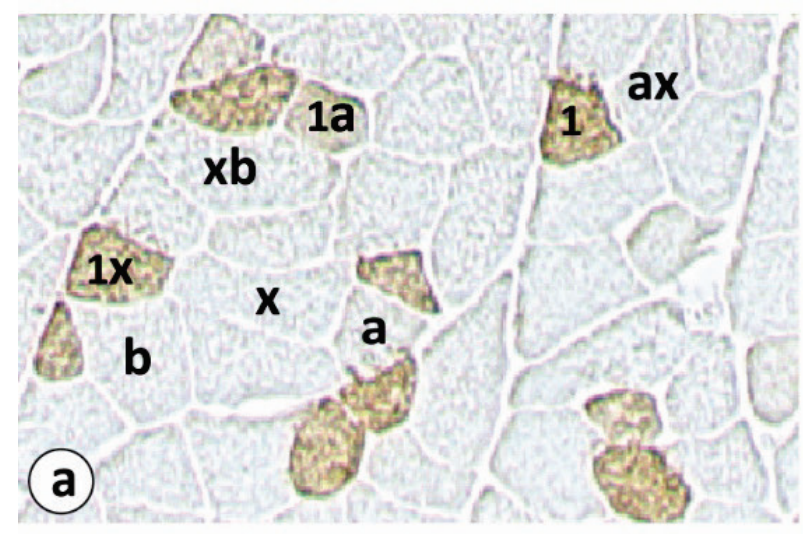

DMH

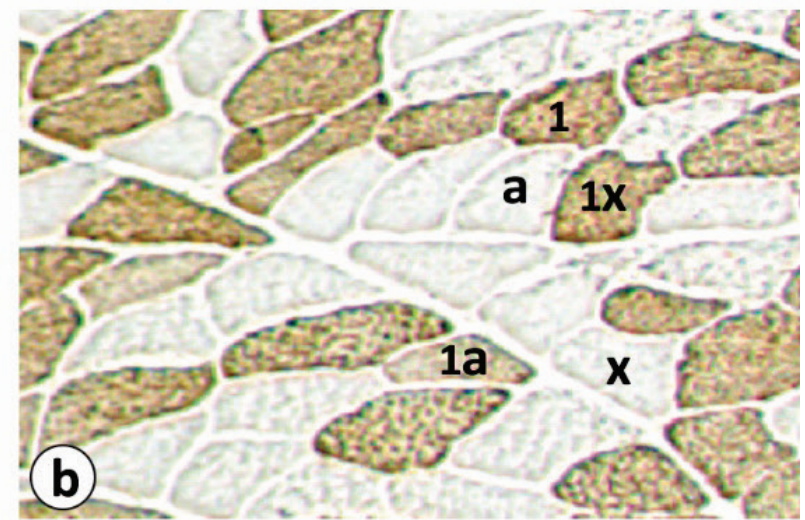

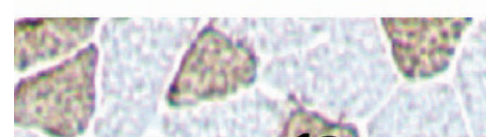

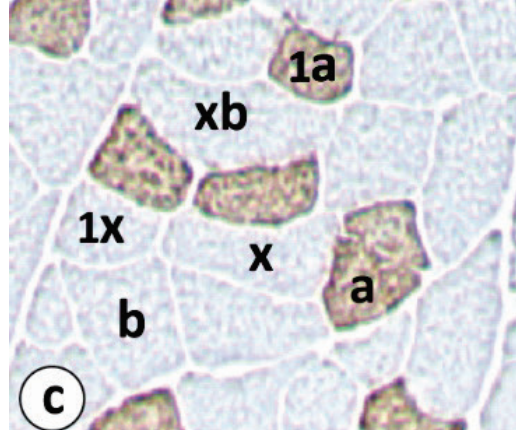

(c) 20
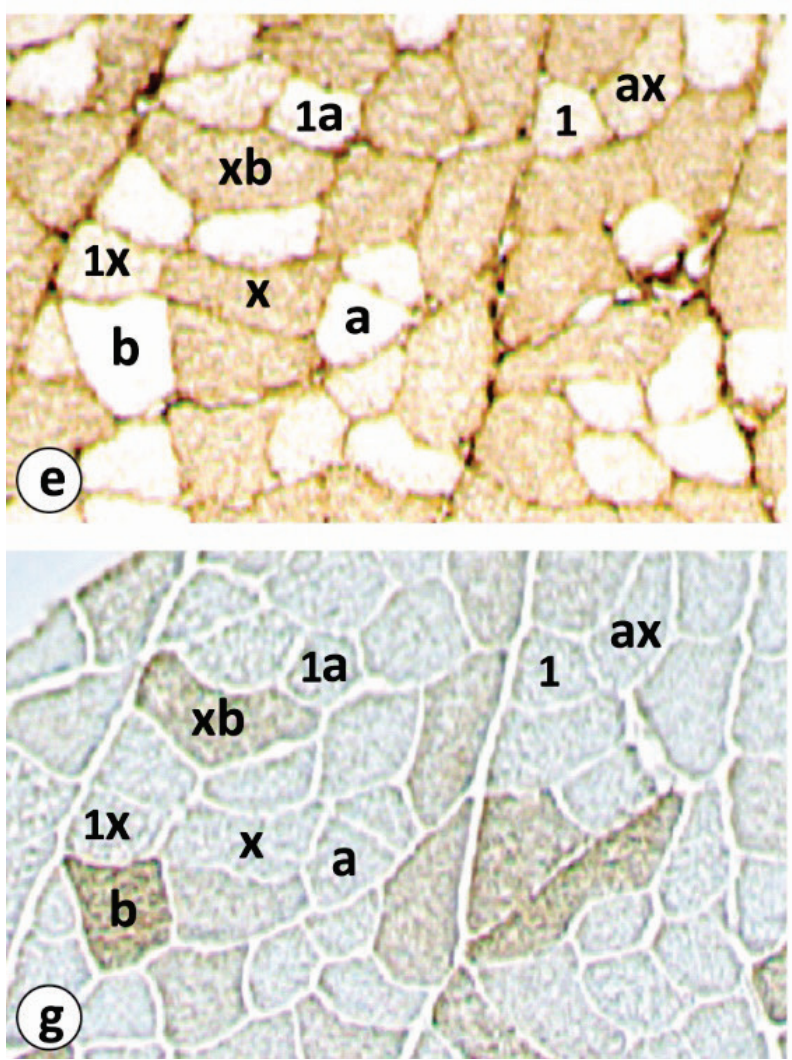

1

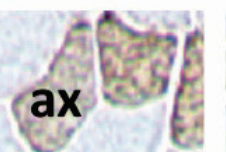

86

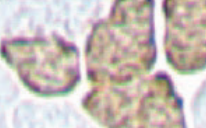

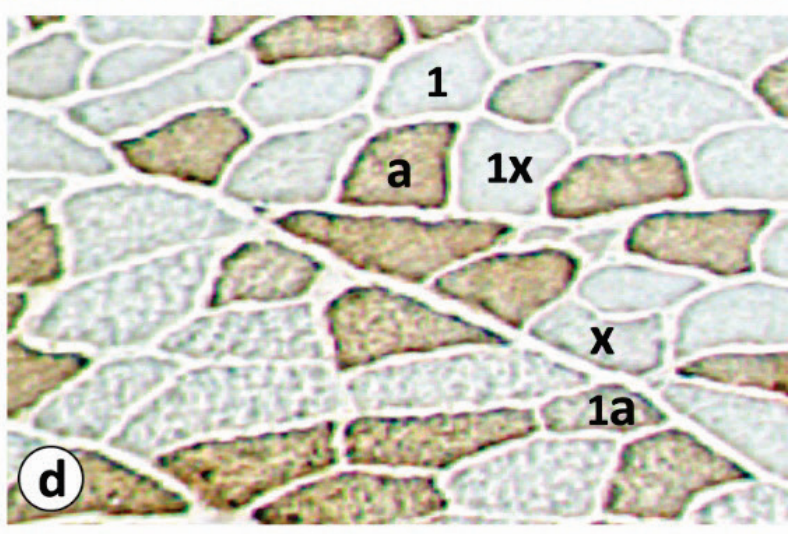

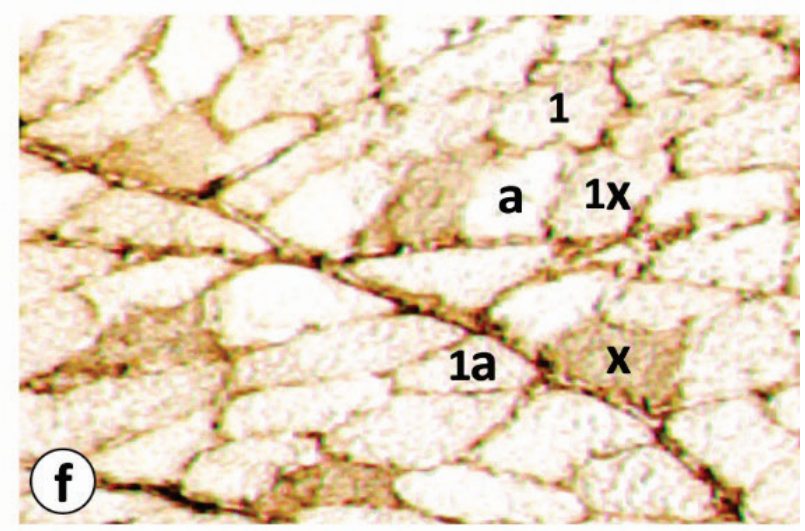

1

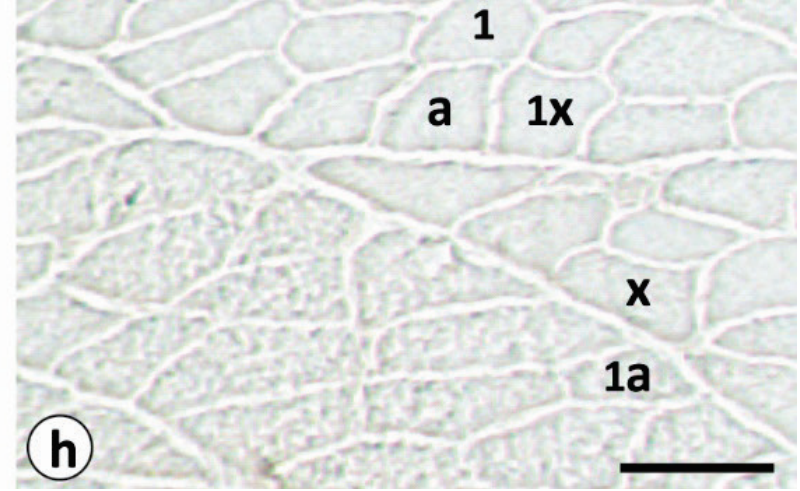

Fig. 1. Fiber types in the deep portion of gastrocnemius lateralis (GLd) muscle of control (a, $\mathbf{c}, \mathbf{e}, \mathbf{g})$ and 1,2-dimethylhydrazine (DMH) treated (DMH-treated) rat $(\mathbf{b}, \mathbf{d}, \mathbf{f}, \mathbf{h})$. The fiber types were immunohistochemically determined according to the MyHC expression with monoclonal antibodies: BA-D5 specific to MyHC-1 (a, b), SC-71 specific to MyHC-2a (c, d), 6H1 specific to MyHC-2x (e, f) and BF-F3 specific to MyHC-2b ( $\mathbf{g}, \mathbf{h})$. Note that individual fiber types are respectively labelled as follows: $1=1,1 / 2 a=1 a, 1 / 2 x=1 x, 2 a=a, 2 a x=a x$, $2 x=x, 2 x b=x b, 2 b=b$. Scale bar $=100 \mu \mathrm{m}$. 


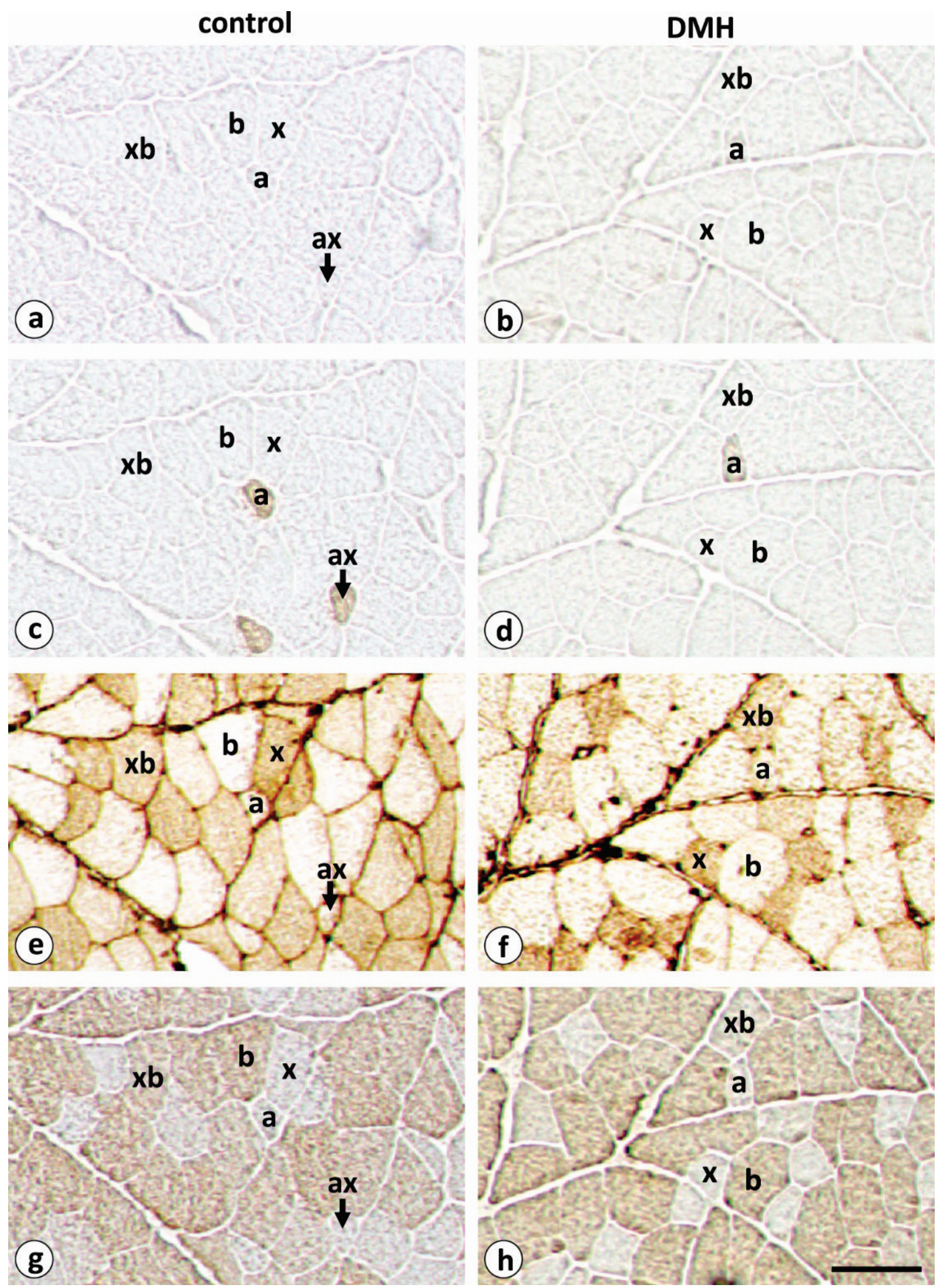

Fig. 2. Fiber types in the superficial portion of gastrocnemius lateralis (GLs) muscle of control $(\mathbf{a}, \mathbf{c}, \mathbf{e}, \mathbf{g})$ and 1,2 -dimethylhydrazine $(\mathrm{DMH})$ treated (DMH-treated) rat $(\mathbf{b}, \mathbf{d}, \mathbf{f}, \mathbf{h})$. The fiber types were immunohistochemically determined according to MyHC expression with monoclonal antibody BA-D5 specific to MyHC-1 (a, b), SC-71 specific to MyHC-2a (c, d), 6H1 specific to MyHC-2x (e, f) and BF-F3 specific to MyHC-2b $(\mathbf{g}, \mathbf{h})$. Note that type 1 fibers were absent and that individual fiber types are respectively labelled as follows: $2 a=a, 2 a x=a x, 2 x=x, 2 x b=x b, 2 b=b$. Scale bar $=100 \mu m$. 

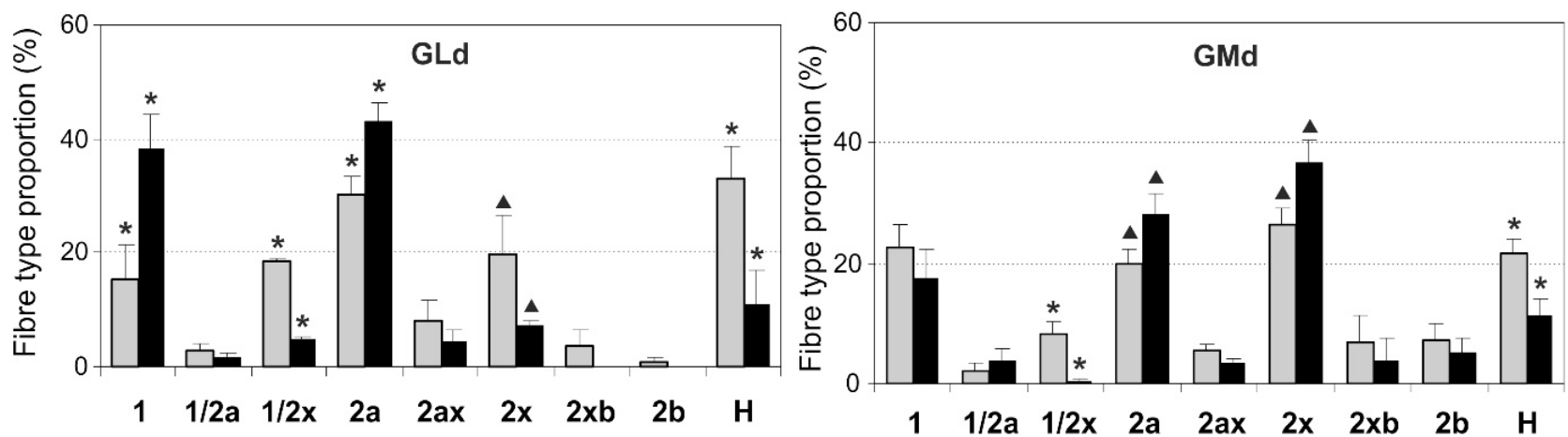

Fibre type
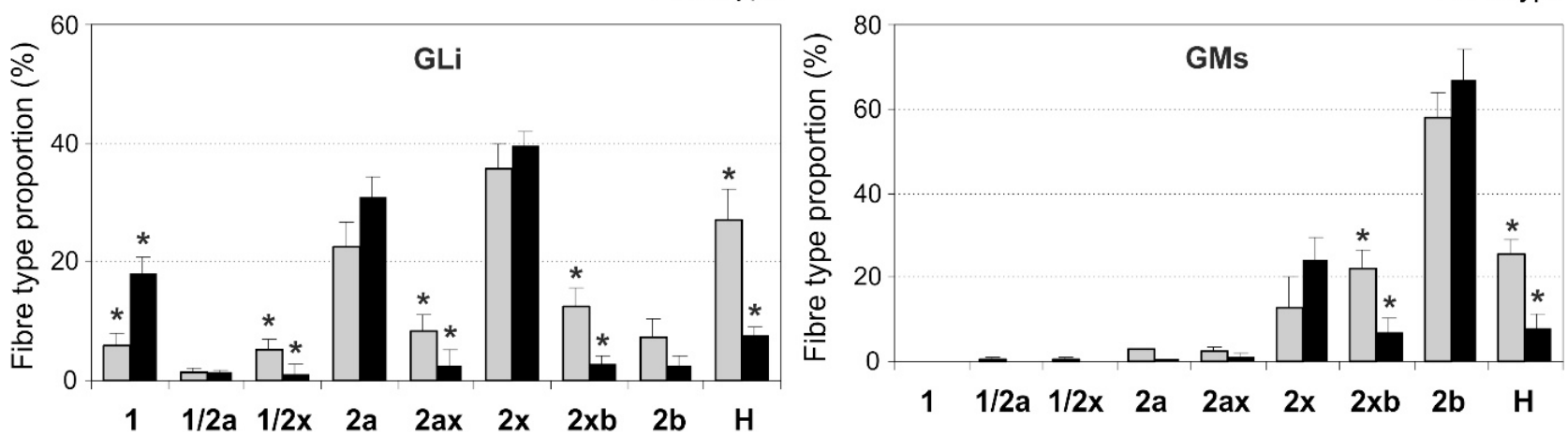

Fibre type
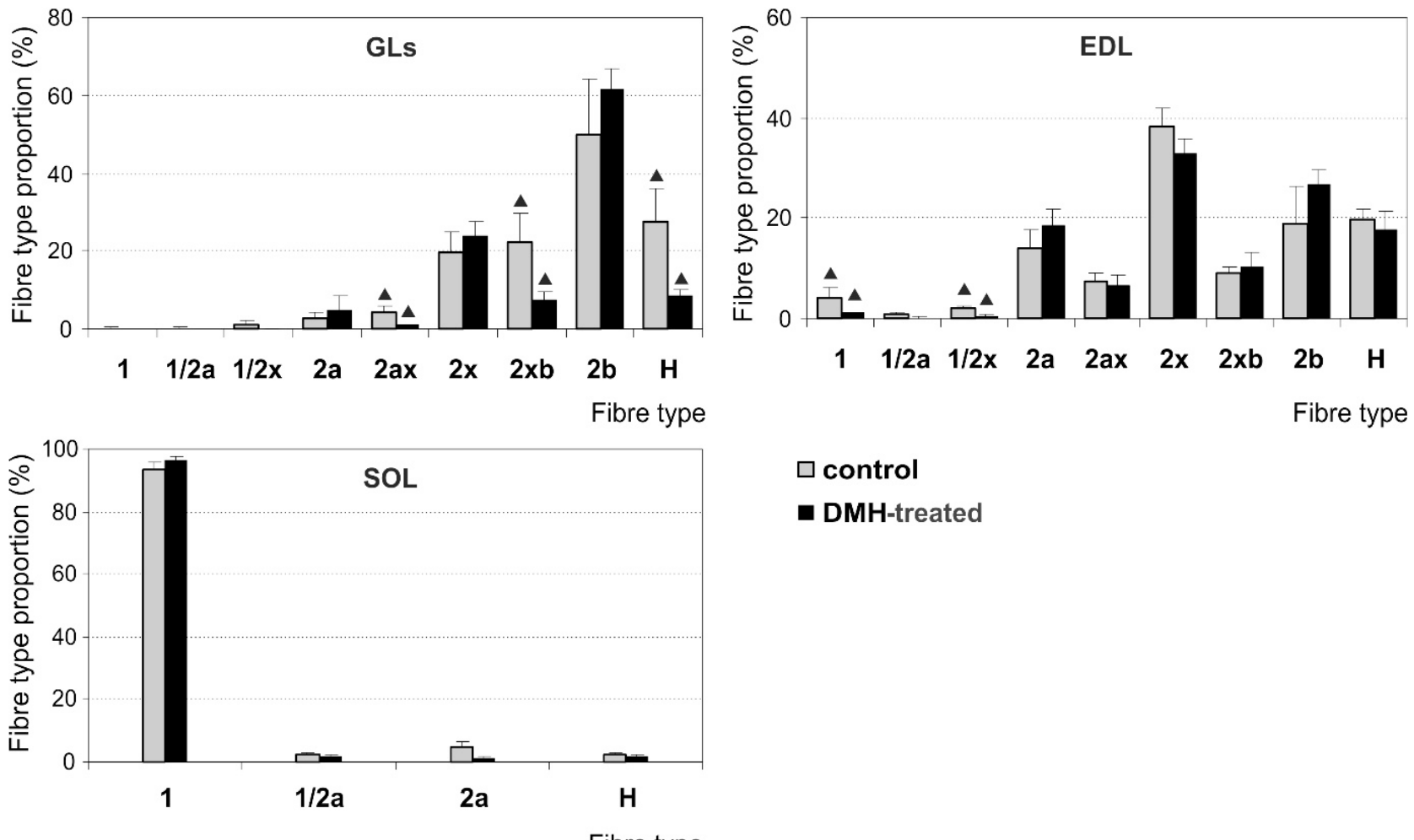

$\square$ control

- DMH-treated

Fig. 3. Average proportions (\%) of fiber types in the muscles of the control $(n=5)$ and 1,2-dimethylhydrazine (DMH) treated rats (DMH-treated, $n=6)$. The average proportions of four pure fiber types $(1,2 a, 2 x$ and $2 b)$, four of the most numerous hybrid fiber types $(1 / 2 a, 1 / 2 x, 2 a x$, and $2 x b)$, and pooled all hybrid fiber types $(H)$ are presented in the deep, intermediate and superficial portion of gastrocnemius lateralis (GLd, GLi, GLs), the deep and superficial portion of gastrocnemius medialis (GMd, GMs), extensor digitorum longus (EDL) and soleus (SOL) muscles. The values are means \pm SEM, significant differences $(p<0.05)$ between the control and DMH-treated rats are labelled by an asterisk $(*)$ and the trends for differences $(p=0.05-0.1)$ are labelled by a triangle $(\boldsymbol{\Lambda})$. 

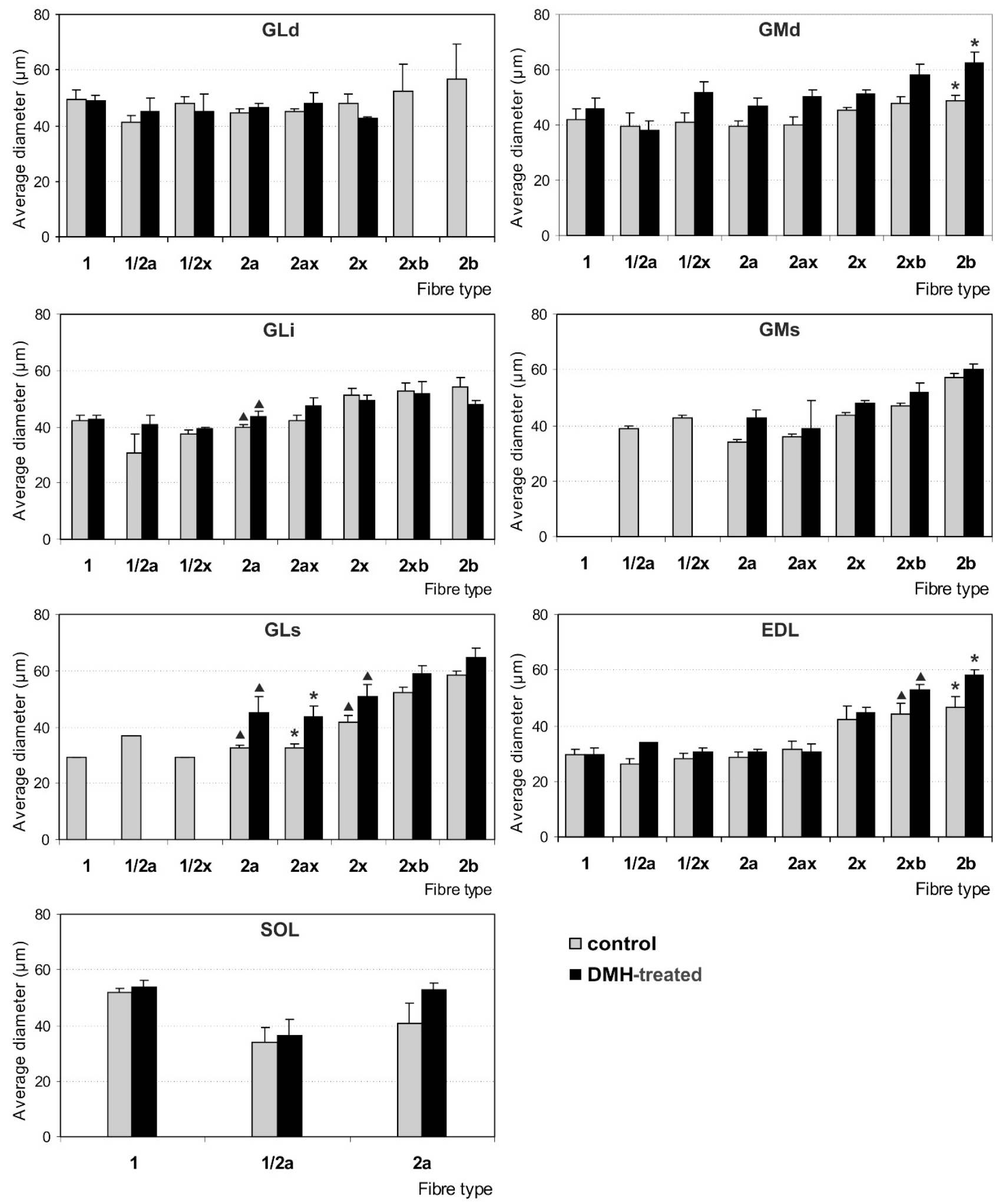

$\square$ control

- DMH-treated

Fig. 4. Average diameters $(\mu \mathrm{m})$ of fiber types $(1,1 / 2 a, 1 / 2 x, 2 a, 2 a x, 2 x, 2 x b$ and $2 b)$ in the extensor digitorum longus (EDL), soleus (SOL), superficial, intermediate, and deep portion of the gastrocnemius lateralis (GLs, GLi, GLd) and the superficial and deep portion of the gastrocnemius medialis (GMs, GMd) muscles of the control and 1,2-dimethylhydrazine (DMH) treated rats (DMH-treated). The values are means \pm SEM, significant differences $(p<0.05)$ between the control and DMH-treated rats are labelled by an asterisk $(*)$ and trends for differences $(p=0.05-0.1)$ are labeled by a triangle $(\boldsymbol{\Lambda})$. 


\section{Fiber type transitions}

DMH treatment induced significant differences in the average proportions of some fiber types in GLd, GLi, GMd and GMs samples only (Fig. 3). The proportion of type 1 and $2 \mathrm{a}$ fibers significantly increased in GLd, whereas that of the hybrid $1 / 2 \mathrm{x}$ fibers significantly decreased (Fig. 1). These results and a trend of type $2 \mathrm{x}$ proportion decrease imply transitions from faster fiber types, especially from type $2 x$, towards slower ones ( 1 and $2 a$ ) in GLd. Similar but less pronounced trend was evident in GLi as well. The proportions of the three most numerous hybrid fiber types, $1 / 2 \mathrm{x}, 2 \mathrm{ax}$, and $2 \mathrm{xb}$, decreased significantly, which had obviously resulted in a significant increase of type 1 and also in a slight increase of type $2 \mathrm{a}$ and $2 \mathrm{x}$ proportions. Like in GLd and GLi, in GMd of DMH-treated rats the proportion of hybrid type $1 / 2 x$ significantly decreased and was accompanied with a trend of type $2 \mathrm{a}$ and $2 \mathrm{x}$ proportions increase implying trends of transitions towards these two fast fiber types. In GMs only the proportion of hybrid type $2 \mathrm{xb}$ and pooled hybrid fibers decreased significantly. To summarize, in all gastrocnemius samples DMH treatment induced either a significant decrease or a trend of pooled hybrid fiber types to decrease (Fig. 3H). On the contrary, in the SOL and EDL muscles of DMH-treated rats there were no significant shifts in the fiber types, while in EDL muscle there was only a trend for the type 1 and $1 / 2 x$ proportion decrease.

\section{Changes in the fiber type size}

To evaluate whether DMH treatment affected the size of the various types of fibers, the average diameters of the four pure and four of the most numerous hybrid fiber types within homonymous muscles of both animal groups were compared (Fig. 4). The average diameters of most fiber types did not differ significantly between the two groups, only those of type $2 b$ in GMd and EDL and that of type 2ax in GLs increased significantly in the DMH-treated group. Nevertheless, the average diameters of most fiber types had tendency to increase in DMH-treated rats, especially those of type $2 \mathrm{a}$ in GLi, type $2 \mathrm{a}$ and $2 \mathrm{x}$ in GLs, and type $2 \mathrm{xb}$ in EDL.

\section{Discussion}

In the present study we have demonstrated that 15-week DMH treatment had only a mild effect on the fiber type transitions in some rat skeletal muscles, but had almost no significant effect on the fiber type size.

Cancerogenesis itself is among the potential factors that affect the skeletal muscle. Namely, in its progressive stage the cancerogenesis is associated with cachexia, muscle wasting, and fiber atrophy, preferentially that of the fastest type 2 fibers in the fast muscles (Acharyya et al. 2005, Ciciliot et al. 2013) due to increased degradation of proteins (Llovera et al. 1994, Temparis et al. 1994, Baracos et al. 1995, Lecker et al. 2004, Acharyya et al. 2005). In our study DMH treatment did not induce fiber atrophy; on the contrary, the average diameters of fiber types had a tendency to increase in DMH-treated rats. Such findings are in accordance with the fact that our experiment was concluded before the rats developed any signs of cachexia or muscle wasting (Perse et al. 2009, Perše et al. 2012).

Our result showed that DMH treatment did not severely affect MyHC isoform expression. Namely, only some significant fiber type transitions, mostly from hybrid towards pure fiber types, were evident in the gastrocnemius samples of DMH-treated rats, but were negligible in SOL and EDL. Such differing responses to DMH treatment across the analyzed muscles are not surprising, since each muscle possesses its own "adaptive range" for fiber type transitions (Schiaffino and Reggiani 2011). The muscle's adaptive capacity is assumed to be related to its original and genetically determined fiber type composition, appropriate to the muscle's functional role and may be also species-specific (Blaauw et al. 2013). However, it can be secondarily modulated by various extrinsic factors such as the type of innervation or electrical stimulation, muscle loading or activity level, and hormonal factors, especially thyroid hormones (Pette and Staron 1997, Baldwin and Haddad 2002, Schiaffino and Reggiani 2011, Blaauw et al. 2013, Soukup and Smerdu 2015).

The most surprising finding of our study was a pronounced transition of hybrid fiber types towards pure ones in gastrocnemius samples of DMH-treated rats. Namely, in the past it was considered that hybrid fibers are the result of pure fiber types undergoing transitions through the shift in $\mathrm{MyHC}$ isoform expression and that the co-expression of $\mathrm{MyHC}$ isoforms occurs due to relatively slow protein turnover, i.e. about 30 days. Thus, they were assumed to be more numerous in muscles under changed physiological conditions (Pette and Staron 1997, Talmadge 2000, Baldwin and Haddad 2001, Schiaffino and Reggiani 2011), as found for instance after denervation (Patterson et al. 2006). Therefore, higher 
proportions of hybrid fiber types would be expected in DMH-treated rats. Nevertheless, our findings are in agreement with more recent reports on numerous hybrid fibers in non-transforming muscles (DeNardi et al. 1993, Staron et al. 1999, Caiozzo et al. 2003, Stephenson 2001, Glaser et al. 2010).

Given that the proportions of the pooled hybrid fiber types decreased in DMH-treated rats, we assume that such a decrease may be the result of kind of "instability" or "readiness" of the hybrid fibers to more rapidly adapt with a shift towards the expression of only one of the primary co-expressed isoforms. For instance, in GLd the decrease in the proportion of $1 / 2 \mathrm{x}$ fibers was accompanied with a substantial increase of type 1 fibers proportion. Similarly, a resistance training in humans resulted in the reduction of hybrid fiber type proportions with concomitant increase of pure fiber type proportions (Williamson et al. 2001). A non-uniform, but musclespecific transition of hybrid fibers towards pure ones was demonstrated in some maturing mouse skeletal muscles as well, but in some muscles (SOL) hybrid fibers persisted (Brummer et al. 2013). Moreover, it was demonstrated that different patterns of exercise not only differently affected the proportions of human hybrid type $1 / 2 \mathrm{a}$ and $2 \mathrm{ax}$ fibers but the proportions of the co-expressed MyHC isoforms within a single hybrid fiber as well (Kohn et al. 2007). These results suggest that the co-expressed MyHC isoforms in a hybrid fiber are under independent regulatory control.

Another unexpected finding was the second most numerous hybrid fiber type $1 / 2 x$, which does not conform to the proposed way for fiber type transitions following the principle of the "next-neighbor" MyHC isoform co-expression $\quad(1 \leftrightarrow 1 / 2 \mathrm{a} \leftrightarrow 2 \mathrm{a} \leftrightarrow 2 \mathrm{ax} \leftrightarrow 2 \mathrm{x} \leftrightarrow$ $2 \mathrm{xb} 2 \mathrm{~b}$ ) (Pette and Staron 1997). Finding quite a lot of $1 / 2 \mathrm{x}$ fibers in GLd $(18.5 \%)$, GMd (8.3\%) and GLi $(5.4 \%)$ samples of control rats, we initially assumed that the antibody specific to MyHC-2x cross-reacted with MyHC-1. Since such staining was neither apparent in all fibers expressing MyHC-1, nor in all muscles, and since $1 / 2 x$ fibers were less numerous in the above-listed muscles of DMH-treated rats, we excluded the crossreactivity. Quite high proportions of $1 / 2 \mathrm{x}$ fibers, demonstrated in the hind limb muscles and diaphragm of Wistar-Kyoto and Sprague-Dawley rats by single fiber electrophoresis (Bortolotto et al. 2000, Caiozzo et al. 2003), imply that in the past their appearance was underestimated in immunohistochemical studies due to the lack of an antibody exclusively specific to MyHC-2x.
Therefore, we assume that $1 / 2 \mathrm{x}$ fibers are not only rapidly transforming, the so called "jump" fibers, skipping the gradual transition as previously suggested (Andersen et al. 1999, Talmadge 2000), but they more likely represent a physiological fiber type, probably possessing specific contractile characteristics. Thus hybrid fibers should be considered not only as transforming fibers but obviously as a normal physiological fiber type as well. The physiological implications of such single fiber polymorphism are not clearly understood. It is assumed that hybrid fiber types represent a physiological continuum of fibers with a continuum of contractile properties, which expand the functional repertoire of a skeletal muscle and also enable smoother fiber type transitions and fine tuning with changed physiological demands.

Analyzing just MyHC isoform expression, we can only speculate about the cellular and molecular mechanisms that could underlay the DMH-induced fiber type transitions. As in the intestine DMH treatment affects various signalling pathways and molecules (Roy et al. 2001, Kolligs et al. 2002, Perše and Cerar 2011), it might affect even those implicated in the regulation of MyHC gene expression. For example, the Wnt/ $\beta$-catenin pathway, which interacts with the other, so-called non-canonical Wnt/calcium pathway (Sugimura and Li 2010, Thrasivoulou et al. 2013), is substantially affected in the colorectal carcinogenesis (Kolligs et al. 2002). The latter is known to regulate intracellular calcium levels (Komiya and Habas 2008), which are one of the main activity correlates that activate the signalling pathways regulating the fiber type transitions (Chin et al. 1998, Crabtree and Schreiber 2009, Gundersen 2011, Schiaffino and Reggiani 2011, Blaauw et al. 2013). DMH treatment also affects peroxisome proliferator-activated receptor $\delta$ (PPARס) (Chung 2000, Takayama et al. 2006), a signaling molecule that is a member of nuclear receptors with multiple functions and is importantly implicated in the regulation of fiber type transitions as well (Mahoney et al. 2005, De Wilde et al. 2008). Another signalling pathway affected in colorectal carcinogenesis is TGF $\beta$ (Chung 2000, Markowitz et al. 1995). This or more precisely the myostatin-Smad $2 / 3$ pathway is assumed to be implicated in the onset of muscle atrophy (Ciciliot et al. 2013, Blaauw et al. 2013, Schiaffino et al. 2013).

Another potential mechanism that might be implicated in DMH-induced fiber type transitions is the central nervous system, since the pattern of neural 
impulses is one of the major factors that determine the muscles' fiber type composition (Schiaffino and Reggiani 2011, Blaauw et al. 2013). Some studies demonstrated that $\mathrm{DMH}$ affected diurnal levels of neurotransmitters (norepinephrine, dopamine, metabolite of serotonin) in hypothalamic nuclei in rats (Arutjunyan et al. 2001) and brain neurotransmitter status by increasing brain epinephrine and decreasing brain serotonin and norepinephrine levels (Pandey et al. 2015). Since increased levels of brain serotonin are associated with increased fatigue (Bailey et al. 1993), increased daily activities of DMH-treated rats with presumably lower level of brain serotonin would be expected. However, long term effects of increased daily activities reflect in various clinical or biochemical characteristics such as caloric intake, body weight, serum lipids profile, etc. Since on one hand Pandey et al. (2015) did not analyze any of these parameters and on the other hand we found no significant differences in various clinical and biochemical parameters (i.e. daily food or caloric intake, body weight gain, abdominal fat, the level of LDH in the serum, the level of corticosterone, relative weight of internal organs, or lipids in the blood) between the control and DMH-treated rats (details are in Perse et al. 2012), further studies are necessary to elucidate whether the changes in the brain neurotransmitters are caused directly by $\mathrm{DMH}$ or are the consequence of the toxic effect of DMH on other organs and tissues. In addition it is also possible that $\mathrm{DMH}$-induced fiber type transitions might result from DMH side effects on the motor system by subtly altering the pattern of nerve impulses to motor units. Nevertheless, further studies are necessary to reveal the cellular and molecular mechanisms underlying the $\mathrm{DMH}$-induced fiber type transitions.

In conclusion, the present study showed that 15-week DMH treatment may affect skeletal muscle fibers to some extent, but, as demonstrated, the effect is muscle-specific and should not be generalized. This findings must be taken into consideration not only when evaluating various skeletal muscle characteristics or alterations in DMH-treated animal models, but also in generally, when evaluating the capacity of skeletal muscles to adapt to changed physiological and pathological conditions.

\section{Conflict of Interest}

There is no conflict of interest.

\section{Acknowledgements}

We thank to Mrs. Nataša Pollak, Mrs. Majda ČrnakMaasarani, Mrs. Andreja Vidmar, Mr. Marko Slak, and Mr. Milan Števanec for their technical assistance. The research was supported by the Slovenian Research Agency (P3-0043 and P3-0054).

\section{References}

ACHARYYA S, BUTCHBACH ME, SAHENK Z, WANG H, SAJI M, CARATHERS M, RINGEL MD, SKIPWORTH RJ, FEARON KC, HOLLINGSWORTH MA, ET AL.: Dystrophin glycoprotein complex dysfunction: a regulatory link between muscular dystrophy and cancer cachexia. Cancer Cell 8: 421-432, 2005.

ANDERSEN JL, GRUSCHY-KNUDSEN T, SANDRI C, LARSSON L, SCHIAFFINO S: Bed rest increases the amount of mismatched fibers in human skeletal muscle. J Appl Physiol (1985) 86: 455-460, 1999.

ARUTJUNYAN AV, KERKESHKO GO, ANISIMOV VN, STEPANOV MG, PROKOPENKO VM, POZDEYEV NV, KORENEVSKY AV: Disturbances of diurnal rhythms of biogenic amines contents in hypothalamic nuclei as an evidence of neurotropic effects of enterotropic carcinogen 1,2-dimethylhydrazine. Neuro Endocrinol Lett 22: 229-237, 2001.

BALDWIN KM, HADDAD F: Effects of different activity and inactivity paradigms on myosin heavy chain gene expression in striated muscle. J Appl Physiol (1985) 90: 345-357, 2001.

BALDWIN KM, HADDAD F: Skeletal muscle plasticity: cellular and molecular responses to altered physical activity paradigms. Am J Phys Med Rehabil 81 (11 Suppl): S40-S51, 2002.

BARACOS VE, DEVIVO C, HOYLE DH, GOLDBERG AL: Activation of the ATP-ubiquitin-proteasome pathway in skeletal muscle of cachectic rats bearing a hepatoma. Am J Physiol 268: E996-E1006, 1995.

BAILEY SP, DAVIS JM, AHLBORN EN: Serotonergic agonists and antagonists affect endurance performance in the rat. Int J Sports Med 14: 330-333, 1993.

BLAAUW B, SCHIAFFINO S, REGGIANI C: Mechanisms modulating skeletal muscle phenotype. Compr Physiol 3: $1645-1687,2013$. 
BORTOLOTTO SK, CELLINI M, STEPHENSON DG, STEPHENSON GM: MHC isoform composition and Ca(2+)or $\operatorname{Sr}(2+)$-activation properties of rat skeletal muscle fibers. Am J Physiol Cell Physiol 279: C1564-C1577, 2000.

BRUCE WR: Counterpoint: From animal models to prevention of colon cancer. Criteria for proceeding from preclinical studies and choice of models for prevention studies. Cancer Epidemiol Biomarkers Prev 12: 401-404, 2003.

BRUMMER H, ZHANG MY, PIDDOUBNY M, MEDLER S: Hybrid fibers transform into distinct fiber types in maturing mouse muscles. Cells Tissues Organs 198: 227-236, 2013.

CAIOZZO V, BAKER M, HUANG K, CHOU H, WU Y, BALDWIN K: Single-fiber myosin heavy chain polymorphism: how many patterns and what proportions? Am J Physiol Regul Integr Comp Physiol 285: R570-R580, 2003.

CHIN ER, OLSON EN, RICHARDSON JA, YANG Q, HUMPHRIES C, SHELTON JM, WU H, ZHU W, BASSEL-DUBY R, WILLIAMS RS: A calcineurin-dependent transcriptional pathway controls skeletal muscle fiber type. Genes Dev 12: 2499-2509, 1998.

CHUNG DC: The genetic basis of colorectal cancer: insights into critical pathways of tumorigenesis. Gastroenterology 119: 854-865, 2000.

CICILIOT S, ROSSI AC, DYAR KA, BLAAUW B, SCHIAFFINO S: Muscle type and fiber type specificity in muscle wasting. Int J Biochem Cell Biol 45: 2191-2199, 2013.

CORPET DE, PIERRE F: How good are rodent models of carcinogenesis in predicting efficacy in humans? A systematic review and meta-analysis of colon chemoprevention in rats, mice and men. Eur J Cancer 41: 1911-1922, 2005.

CRABTREE GR, SCHREIBER SL: SnapShot: Ca2+-calcineurin-NFAT signaling. Cell 138: 210-210.e1, 2009.

DE WILDE J, MOHREN R, VAN DEN BERG S, BOEKSCHOTEN M, DIJK KW, DE GROOT P, MÜLLER M, MARIMAN E, SMIT E: Short-term high fat-feeding results in morphological and metabolic adaptations in the skeletal muscle of C57BL/6J mice. Physiol Genomics 32: 360-369, 2008.

DENARDI C, AUSONI S, MORETTI P, GORZA L, VELLECA M, BUCKINGHAM M, SCHIAFFINO S: Type $2 \mathrm{X}$-myosin heavy chain is coded by a muscle fiber type-specific and developmentally regulated gene. $J$ Cell Biol 123: 823-835, 1993.

DEVASENA T, MENON VP, RAJASEKHARAN KN: Prevention of 1,2-dimethylhydrazine-induced circulatory oxidative stress by bis-1,7-(2-hydroxyphenyl)-hepta-1,6-diene-3,5-dione during colon carcinogenesis. Pharmacol Rep 58: 229-235, 2006.

DRZYMALA-CELICHOWSKA H, KAROLCZAK J, REDOWICZ MJ, BUKOWSKA D: The content of myosin heavy chains in hindlimb muscles of female and male rats. J Physiol Pharmacol 63: 187-193, 2012.

GLASER BW, YOU G, ZHANG M, MEDLER S: Relative proportions of hybrid fibers are unaffected by 6 weeks of running exercise in mouse skeletal muscles. Exp Physiol 95: 211-221, 2010.

GORZA L: Identification of a novel type 2 fiber population in mammalian skeletal muscle by combined use of histochemical myosin ATPase and anti-myosin monoclonal antibodies. J Histochem Cytochem 38: 257-265, 1990.

GUNDERSEN K: Excitation-transcription coupling in skeletal muscle: the molecular pathways of exercise. Biol Rev Camb Philos Soc 86: 564-600, 2011.

KAREN P, STEVANEC M, SMERDU V, CVETKO E, KUBÍNOVÁ L, ERZEN I: Software for muscle fiber type classification and analysis. Eur J Histochem 53: 87-95, 2009.

KOBAEK-LARSEN M, THORUP I, DIEDERICHSEN A, FENGER C, HOITINGA MR: Review of colorectal cancer and its metastases in rodent models: comparative aspects with those in humans. Comp Med 50: 16-26, 2000.

KOHN TA, ESSEN-GUSTAVSSON B, MYBURGH KH: Exercise pattern influences skeletal muscle hybrid fibers of runners and nonrunners. Med Sci Sports Exerc 39: 1977-1984, 2007.

KOLLIGS FT, BOMMER G, GÖKE B: Wnt/beta-catenin/tcf signaling: a critical pathway in gastrointestinal tumorigenesis. Digestion 66: 131-144, 2002.

KOMIYA Y, HABAS R: Wnt signal transduction pathways. Organogenesis 4: 68-75, 2008. 
LECKER SH, JAGOE RT, GILBERT A, GOMES M, BARACOS V, BAILEY J, PRICE SR, MITCH WE, GOLDBERG AL: Multiple types of skeletal muscle atrophy involve a common program of changes in gene expression. FASEB J 18: 39-51, 2004.

LLOVERA M, GARCÍA-MARTÍNEZ C, AGELL N, MARZÁBAL M, LÓPEZ-SORIANO FJ, ARGILÉS JM: Ubiquitin gene expression is increased in skeletal muscle of tumour-bearing rats. FEBS Lett 338: 311-318, 1994.

LUCAS CA, KANG LH, HOH JF: Monospecific antibodies against the three mammalian fast limb myosin heavy chains. Biochem Biophys Res Commun 272: 303-308, 2000.

LUO Y, YONEDA J, OHMORI H, SASAKI T, SHIMBO K, ETO S, KATO Y, MIYANO H, KOBAYASHI T, SASAHIRA T, CHIHARA Y, KUNIYASU H: Cancer usurps skeletal muscle as an energy repository. Cancer Res 74: 330-340, 2014.

MAHONEY DJ, PARISE G, MELOV S, SAFDAR A, TARNOPOLSKY MA: Analysis of global mRNA expression in human skeletal muscle during recovery from endurance exercise. FASEB J 19: 1498-1500, 2005.

MARKOWITZ S, WANG J, MYEROFF L, PARSONS R, SUN L, LUTTERBAUGH J, FAN RS, ZBOROWSKA E, KINZLER KW, VOGELSTEIN B: Inactivation of the type II TGF-beta receptor in colon cancer cells with microsatellite instability. Science 268: 1336-1338, 1995

MATSAKAS A, BOZZO C, CACCIANI N, CALIARO F, REGGIANI C, MASCARELLO F, PATRUNO M: Effect of swimming on myostatin expression in white and red gastrocnemius muscle and in cardiac muscle of rats. Exp Physiol 91: 983-994, 2006.

NETTO LE, RAMAKRISHNA NV, KOLAR C, CAVALIERI EL, ROGAN EG, LAWSON TA, AUGUSTO O: Identification of C8-methylguanine in the hydrolysates of DNA from rats administered 1,2-dimethylhydrazine. Evidence for in vivo DNA alkylation by methyl radicals. J Biol Chem 267: 21524-21527, 1992.

NIELSEN S, PEDERSEN BK: Skeletal muscle as an immunogenic organ. Curr Opin Pharmacol 8: 346-351, 2008.

PANDEY S, SINGH A, CHAUDHARI N, NAMPOOTHIRI LP, KUMAR GN: Protection against 1,2-di-methylhydrazine-induced systemic oxidative stress and altered brain neurotransmitter status by probiotic Escherichia coli CFR 16 secreting pyrroloquinoline quinone. Curr Microbiol 70: 690-697, 2015.

PATTERSON MF, STEPHENSON GM, STEPHENSON DG: Denervation produces different single fiber phenotypes in fast- and slow-twitch hindlimb muscles of the rat. Am J Physiol Cell Physiol 291: C518-C528, 2006.

PEDERSEN BK, AKERSTRÖM TC, NIELSEN AR, FISCHER CP: Role of myokines in exercise and metabolism. J Appl Physiol 103: 1093-1098, 2007.

PERSE M, CERAR A: The dimethylhydrazine induced colorectal tumours in rat - experimental colorectal carcinogenesis. Radiol Oncol 39: 61-70, 2005.

PERSE M, INJAC R, STRUKELJ B, CERAR A: Effects of high-fat mixed-lipid diet and exercise on the antioxidant system in skeletal and cardiac muscles of rats with colon carcinoma. Pharmacol Rep 61: 909-916, 2009.

PERŠE M, CERAR A: Morphological and molecular alterations in 1,2 dimethylhydrazine and azoxymethane induced colon carcinogenesis in rats. J Biomed Biotechnol 2011: 473964, 2011.

PERŠE M, INJAC R, ŠTRUKELJ B, CERAR A: High fat mixed lipid diet modifies protective effects of exercise on 1,2 dimethylhydrazine induced colon cancer in rats. Technol Cancer Res Treat 11: 289-299, 2012.

PETTE D, STARON RS: Mammalian skeletal muscle fiber type transitions. Int Rev Cytol 170: 143-223, 1997.

ROY HK, KAROLSKI WJ, RATASHAK A: Distal bowel selectivity in the chemoprevention of experimental colon carcinogenesis by the non-steroidal anti-inflammatory drug nabumetone. Int J Cancer 92: 609-615, 2001.

SCHIAFFINO S, DYAR KA, CICILIOT S, BLAAUW B, SANDRI M: Mechanisms regulating skeletal muscle growth and atrophy. FEBS J 280: 4294-4314, 2013.

SCHIAFFINO S, SAGGIN L, VIEL A, AUSONI S, SARTORE S, GORZA L: Muscle fiber types identified by monoclonal antibodies to myosin heavy chains. In: Biochemical Aspects of Physical Exercise. BENZI GL, PACKER N, SILIPRANDI N (eds), Elsevier, Amsterdam, 1986, pp 27-34.

SCHIAFFINO S, REGGIANI C: Fiber types in mammalian skeletal muscles. Physiol Rev 91: 1447-1531, 2011.

SHETYE J, MATHIESEN T, FAGERBERG J, RUBIO C: Ear tumours induced by experimental carcinogenesis in the rat: excision prevents early death. Int J Colorectal Dis 9: 125-127, 1994. 
SMERDU V, SOUKUP T: Demonstration of myosin heavy chain isoforms in rat and humans: the specificity of seven available monoclonal antibodies used in immunohistochemical and immunoblotting methods. Eur J Histochem 52: 179-190, 2008.

SOUKUP T, SMERDU V: Effect of altered innervation and thyroid hormones on myosin heavy chain expression and fiber type transitions: a mini-review. Histochem Cell Biol 143: 123-130, 2015.

STARON RS, KRAEMER WJ, HIKIDA RS, FRY AC, MURRAY JD, CAMPOS GE: Fiber type composition of four hindlimb muscles of adult Fisher 344 rats. Histochem Cell Biol 111: 117-123, 1999.

STEPHENSON GM: Hybrid skeletal muscle fibers: a rare or common phenomenon? Clin Exp Pharmacol Physiol 28: 692-702, 2001.

SUGIMURA R, LI L: Noncanonical Wnt signaling in vertebrate development, stem cells, and diseases. Birth Defects Res C Embryo Today 90: 243-256, 2010.

TAKAYAMA O, YAMAMOTO H, DAMDINSUREN B, SUGITA Y, NGAN CY, XU X, TSUJINO T, TAKEMASA I, IKEDA M, SEKIMOTO M, MATSUURA N, MONDEN M: Expression of PPARdelta in multistage carcinogenesis of the colorectum: implications of malignant cancer morphology. Br J Cancer 95: 889-895, 2006.

TALMADGE R: Myosin heavy chain isoform expression following reduced neuromuscular activity: Potential regulatory mechanisms. Muscle Nerve 23: 661-679, 2000.

TEMPARIS S, ASENSI M, TAILLANDIER D, AUROUSSEAU E, LARBAUD D, OBLED A, BÉCHET D, FERRARA M, ESTRELA JM, ATTAIX D: Increased ATP-ubiquitin-dependent proteolysis in skeletal muscles of tumor-bearing rats. Cancer Res 54: 5568-5573, 1994.

THRASIVOULOU C, MILLAR M, AHMED A: Activation of intracellular calcium by multiple Wnt ligands and translocation of $\beta$-catenin into the nucleus: a convergent model of $\mathrm{Wnt} / \mathrm{Ca} 2+$ and $\mathrm{Wnt} / \beta$-catenin pathways. J Biol Chem 288: 35651-35659, 2013.

WILLIAMSON DL, GALLAGHER PM, CARROLL CC, RAUE U, TRAPPE SW: Reduction in hybrid single muscle fiber proportions with resistance training in humans. J Appl Physiol 91: 1955-1961, 2001. 